\title{
Binding of Formic Acid on Anatase $\mathrm{TiO}_{2}(101)$
}

\author{
Yang Wang, ${ }^{\dagger}$ Bo Wen, ${ }^{\ddagger}$ Arjun Dahal, ${ }^{+, \perp}$ Greg A. Kimmel, ${ }^{+}$ \\ Roger Rousseau, ${ }^{\dagger}$ Annabella Selloni,,${ }^{*}$ Nikolay G. Petrik,,${ }^{*}$ and Zdenek Dohnálekt,,${ }^{*}$ \\ † Physical and Computational Sciences Directorate and Institute for Integrated \\ Catalysis, Pacific Northwest National Laboratory, P.O. Box 999, Richland, Washington \\ 99352, United States \\ ‡ Department of Chemistry, Princeton University, Princeton, New Jersey 08544, United \\ States
}

$\S$ Voiland School of Chemical Engineering and Bioengineering, Washington State University, Pullman, Washington 99163, United States

* Indicates the corresponding authors

${ }^{\perp}$ Current Address: Department of Physics, University of South Alabama, Mobile, AL 36688, USA 


\section{Table of Contents}

Section S1. Sample Orientation for TPD and IRAS Measurements..........................................

Section S2. TPD Measurements of the Saturation Coverage of Formic Acid on $\mathrm{Ti}_{5 \mathrm{c}} \mathrm{Sites}_{\text {..........4 }}$

Section S3. Calculations of Gas-Phase Monomer and Dimer Structures ......................................5

Section S4. Imaging Induced Conversion of the Nearest-Neighbor FA Pair to Second-Nearest-

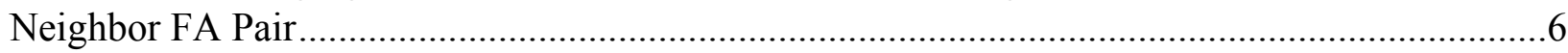

Section S5. Structures and Adsorption Energies Calculated for FA Different Pairs .....................7

Section S6. Comparison of IRAS Spectra of HCOOH and DCOOD Isotopologues ....................8

Section S7. Dose Temperature-Dependent IRAS Spectra …...................................................

Section S8. Annealing Induced Development of Ordered Saturation Layer ................................11 

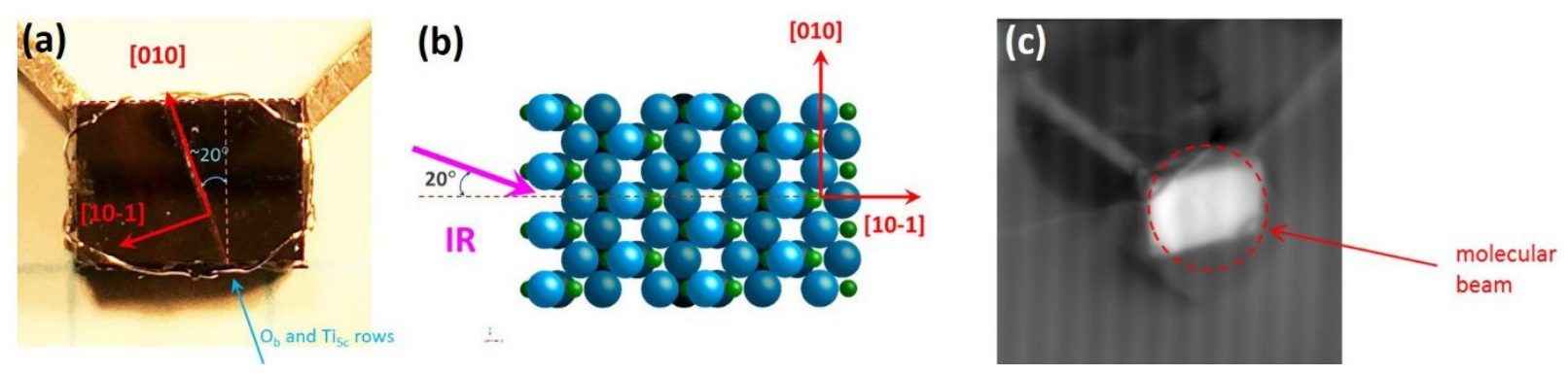

(d)

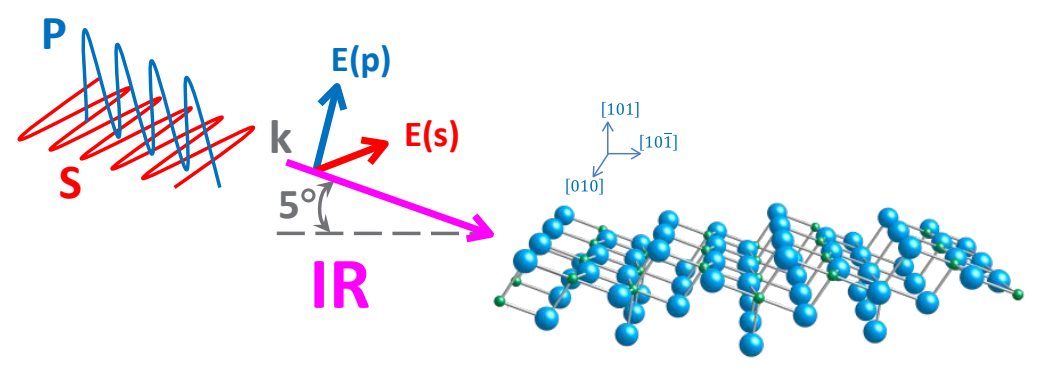

Figure S1. (a) Anatase $\mathrm{TiO}_{2}(101)$ sample with size $\left(7 \times 5 \times 2 \mathrm{~mm}^{3}\right)$ mounted on a tantalum plate holder using Aremco cement and thin tantalum wires at the corners. (b) The IR beam comes normally to the shorter $(5 \mathrm{~mm})$ side of the sample at $20^{\circ}$ to the $[10 \overline{1}]$ azimuth at glancing angle to the (101) surface $\left(5^{\circ}\right)$. (c) Secondary electron image of the sample with a dosed $\mathrm{H}_{2} \mathrm{O}$ film (white spot). The molecular beam $\varnothing 7.3 \mathrm{~mm}$ (umbra), centered on the sample and larger than the sample. d) Schematic for IRAS geometry on $\mathrm{TiO}_{2}(101)$. For s-polarized light, the electric field, $\mathrm{E}(\mathrm{s})$, is parallel to the (101) surface and perpendicular to the IR beam direction shown in (b). For the geometry shown, s-polarized spectra are sensitive to vibrations with a transition dipole moment that has a component parallel to surface and perpendicular to the direction of the IR beam. For p-polarized light, the electric field, E(p), has components normal and parallel to the surface. Thus p-polarized spectra are sensitive to transition dipole moments perpendicular to the (101) surface and parallel to the surface in the direction of the IR beam. 
Section S2. TPD Measurements of the Saturation Coverage of Formic Acid on $\mathrm{Ti}_{5 \mathrm{c}}$ Sites
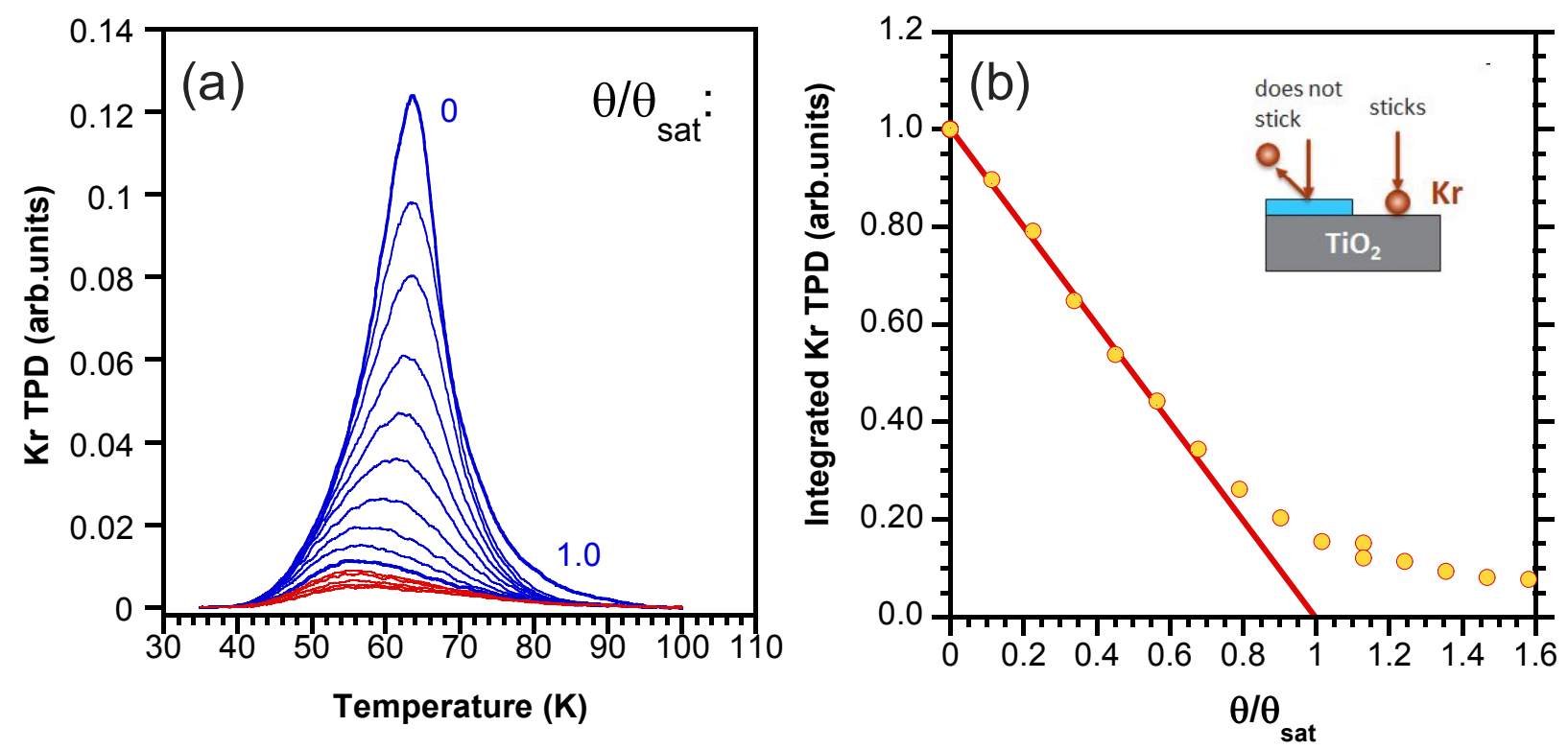

Figure S2. a) Kr TPD spectra for various coverages of the predosed formic acid $\theta / \theta_{\text {sat }}$ (normalized to the saturating dose of formic acid). At the sample base temperature ( $\sim 34 \mathrm{~K}), \mathrm{Kr}$ atoms only stick to the $\mathrm{Ti}_{5 \mathrm{c}}$ sites. When the formic acid is predosed at $80 \mathrm{~K}$, less $\mathrm{Ti}_{5 \mathrm{c}}$ sites are available for the $\mathrm{Kr}$ to adsorb. b) Integrated Kr TPD versus the normalized dose of the formic acid. Linear extrapolation of the initial part of the data set gives the saturating dose of the $\mathrm{HCOOH}$, represented by the combination of mono-dentate and bridging forms of formic acid. From the STM data, $2 \mathrm{HCOOH}$ molecules cover $3 \mathrm{Ti}_{5 \mathrm{c}}$ sites, therefore the absolute coverage at saturation will be $2 / 3 \mathrm{ML} \sim 3.5 \times 10^{14} \mathrm{~cm}^{-2}$. 


\section{Section S3. Calculations of Gas-Phase Monomer and Dimer Structures}

To assess the performance of SCAN functional on the gas phase $\mathrm{HCOOH}$ molecule and $(\mathrm{HCOOH})_{2}$ dimer. Of the two inequivalent, trans- $\mathrm{HCOOH}$ and cis- $\mathrm{HCOOH}$, configurations (Figure S3), trans- $\mathrm{HCOOH}$ is $4.00 \mathrm{kcal} / \mathrm{mol}$ more favorable in energy. This energy difference agrees very well with the $\mathrm{CCSD}(\mathrm{T}) / \mathrm{MP} 2$ result of $4.54 \mathrm{kcal} / \mathrm{mol} .^{3}$ The binding energy of the $\mathrm{HCOOH}$ dimer predicted by SCAN, $-0.8 \mathrm{eV}$, is slightly overestimated compared with the theoretical and experimental values of $-0.5 \mathrm{eV}$ (Ref. ${ }^{3}$ ) and $-0.6 \mathrm{eV}$ (Ref. 2), respectively. Nonetheless, the dimer geometry given by SCAN agrees well with the experiment and high level calculations. ${ }^{1}$

\section{trans-HCOOH}

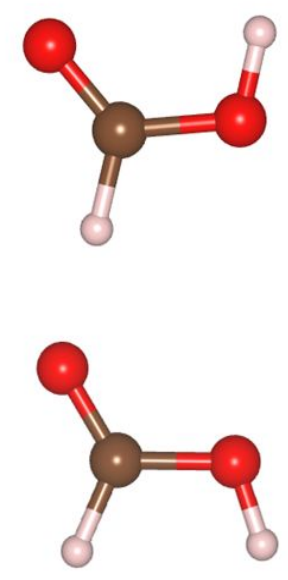

cis-HCOOH

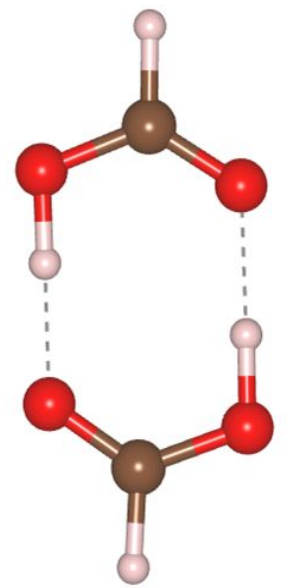

HCOOH-dimer

Figure S3. Configurations of single $\mathrm{HCOOH}$, trans- $\mathrm{HCOOH}$ dimer and single molecule adsorption on anatase (101) surface.

Table 1 SCAN optimized geometries of trans- $\mathrm{HCOOH}$ dimer (distances in $\AA$ and angles in degrees)

\begin{tabular}{ccccccc}
\hline Distances & $\mathrm{C}=\mathrm{O}$ & $\mathrm{C}-\mathrm{O}$ & $\mathrm{O} \cdots \mathrm{O}$ & $\mathrm{C}-\mathrm{H}$ & $\mathrm{O}-\mathrm{H}$ & $\mathrm{H} \cdots \mathrm{O}$ \\
Values & 1.219 & 1.301 & 2.597 & 1.093 & 1.012 & 1.584 \\
Angles & $\angle \mathrm{O}=\mathrm{C}-\mathrm{O}$ & $\angle \mathrm{H}-\mathrm{C}=\mathrm{O}$ & $\angle \mathrm{H}-\mathrm{C}-\mathrm{O}$ & $\angle \mathrm{C}-\mathrm{O}-\mathrm{H}$ & $\angle \mathrm{C}-\mathrm{O} \cdots \mathrm{O}$ & $\angle \mathrm{O}-\mathrm{H} \cdots \mathrm{O}$ \\
Values & 126.12 & 121.48 & 112.4 & 109.14 & 108.86 & 179.11 \\
\hline
\end{tabular}


Section S4. Imaging Induced Conversion of the Nearest-Neighbor FA Pair to SecondNearest-Neighbor FA Pair
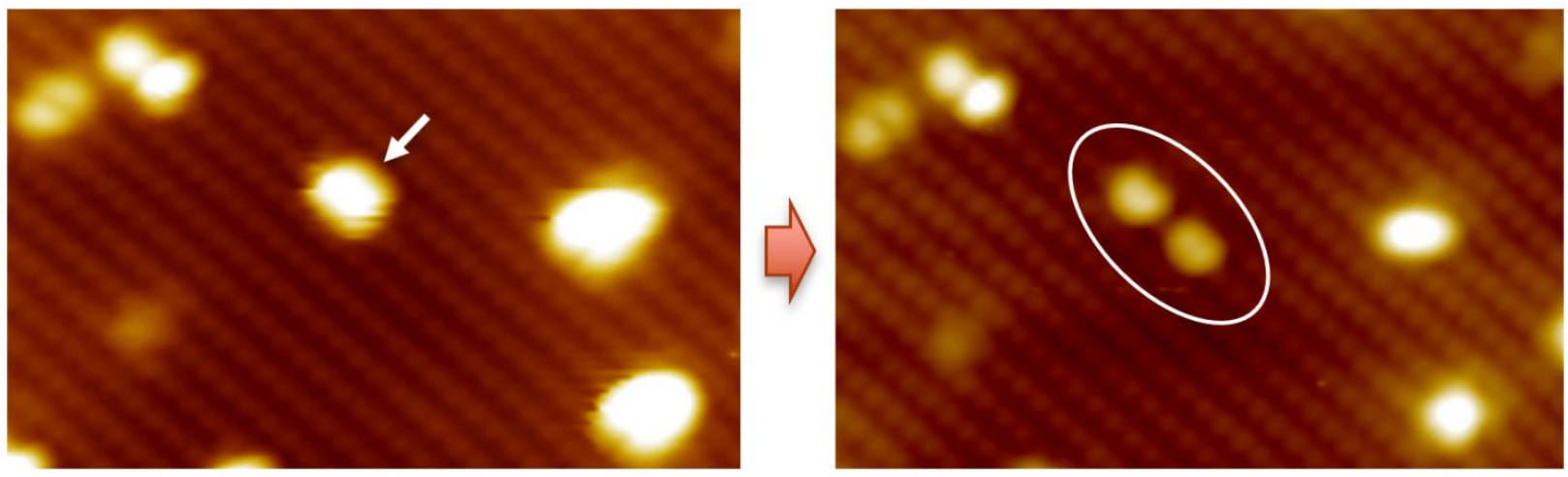

Figure S4. Consecutive STM images demonstrating splitting of the FA nearest-neighbor pair (arrow) during scanning. Only a small fraction of the features are present in this configuration. 
Section S5. Structures and Adsorption Energies Calculated for FA Different Pairs

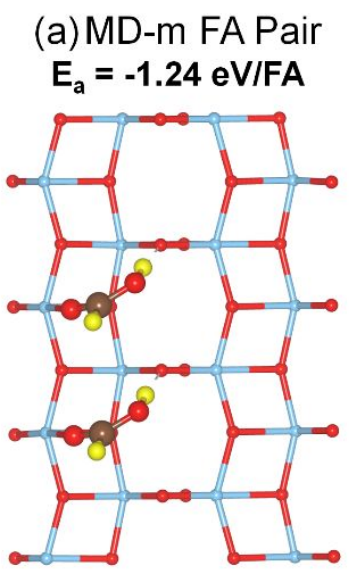

(d) MD-m FA Pair

$E_{\mathrm{a}}=-1.23 \mathrm{eV} / \mathrm{FA}$

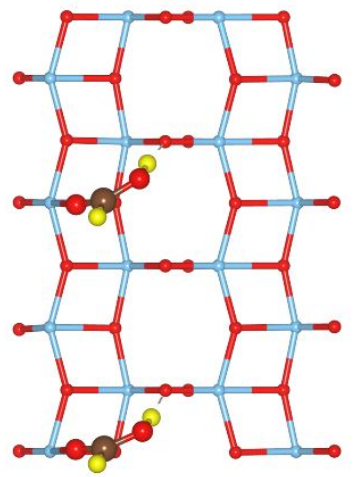

(g) MD-m FA Pair

$$
E_{a}=-1.23 \text { eV/FA }
$$

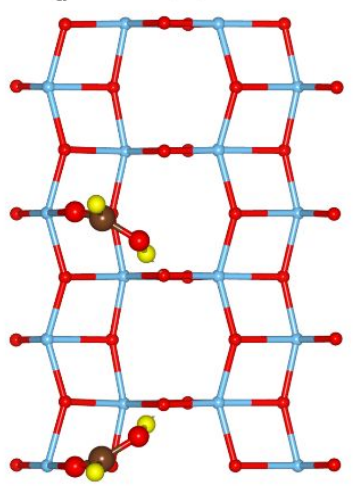

(b) MD-d FA Pair

$E_{a}=-1.14$ eV/FA

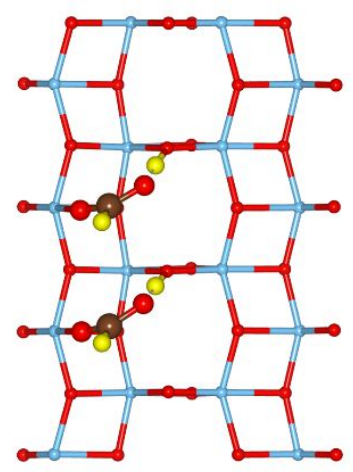

(e) MD-d FA Pair

$E_{a}=-1.13$ eV/FA

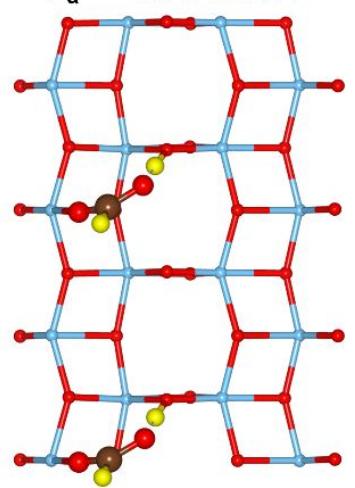

(h) MD-mix FA Pair

$$
E_{a}=-1.18 \text { eV/FA }
$$

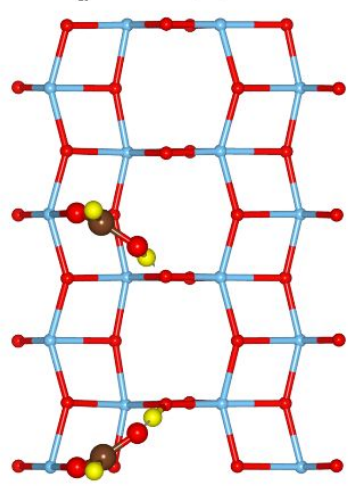

(c) BD FA pair

$E_{\mathrm{a}}=-\mathbf{1 . 3 9} \mathrm{eV} / \mathrm{FA}$

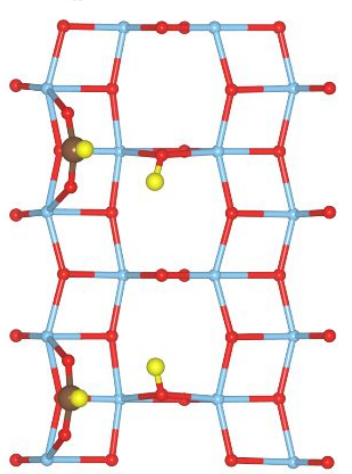

(f) BD FA pair

$E_{a}=-1.38 \mathrm{eV} / F A$

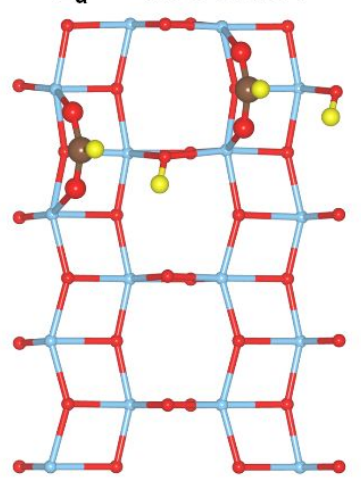

(i) MD-d FA pair

$E_{a}=-1.12$ eV/FA

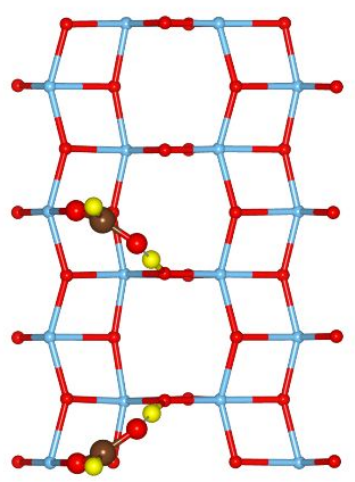

Figure S5. Computed adsorption structures and energies (in $\mathrm{eV}$ ) of different FA pairs on anatase (101): (a) a molecular nearest-neighbor (NN) MD pair; (b) dissociated NN MD pair; (c) a dissociated second-nearest-neighbor (SNN) BD pair; (d) molecular SNN MD pair; (e)

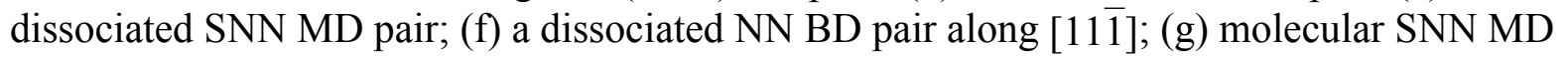
pair with mirror symmetry (MS); (h) mixed molecular-dissociated SNN MD pair with MS; (i) dissociated SNN MD pair with MS. 


\section{Section S6. Comparison of IRAS Spectra of HCOOH and DCOOD Isotopologues}
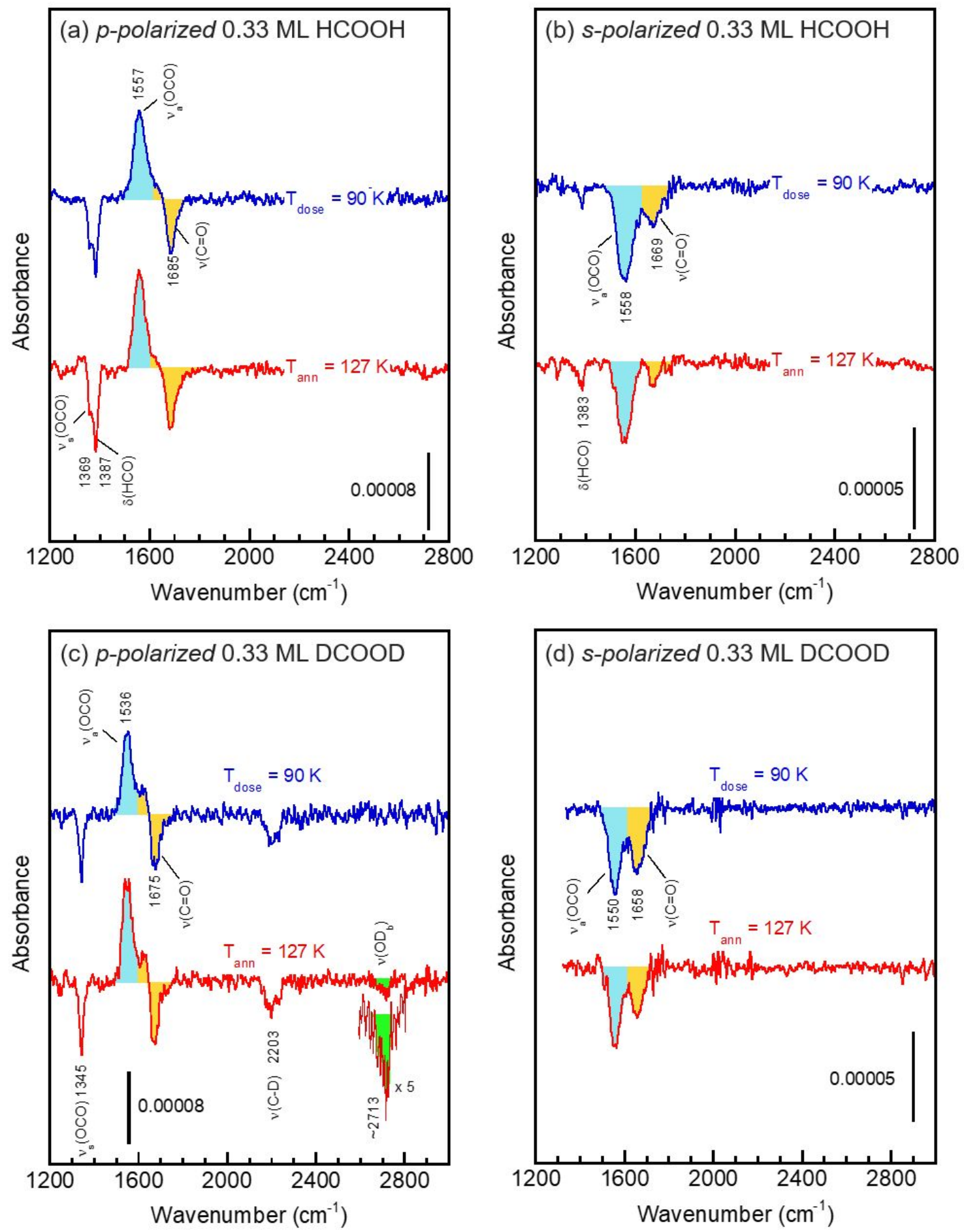

Figure S6. IRAS spectra in p-polarized (a, c) and s-polarized (b, d) modes of 0.33 ML $\mathrm{HCOOH}$ (a, b) and DCOOD (c, d) adsorbed on $\mathrm{TiO}_{2}(101)$ at $90 \mathrm{~K}$ (blue) and subsequently flashed to 127 $\mathrm{K}$ (red). 


\section{Section S7. Dose Temperature-Dependent IRAS Spectra}
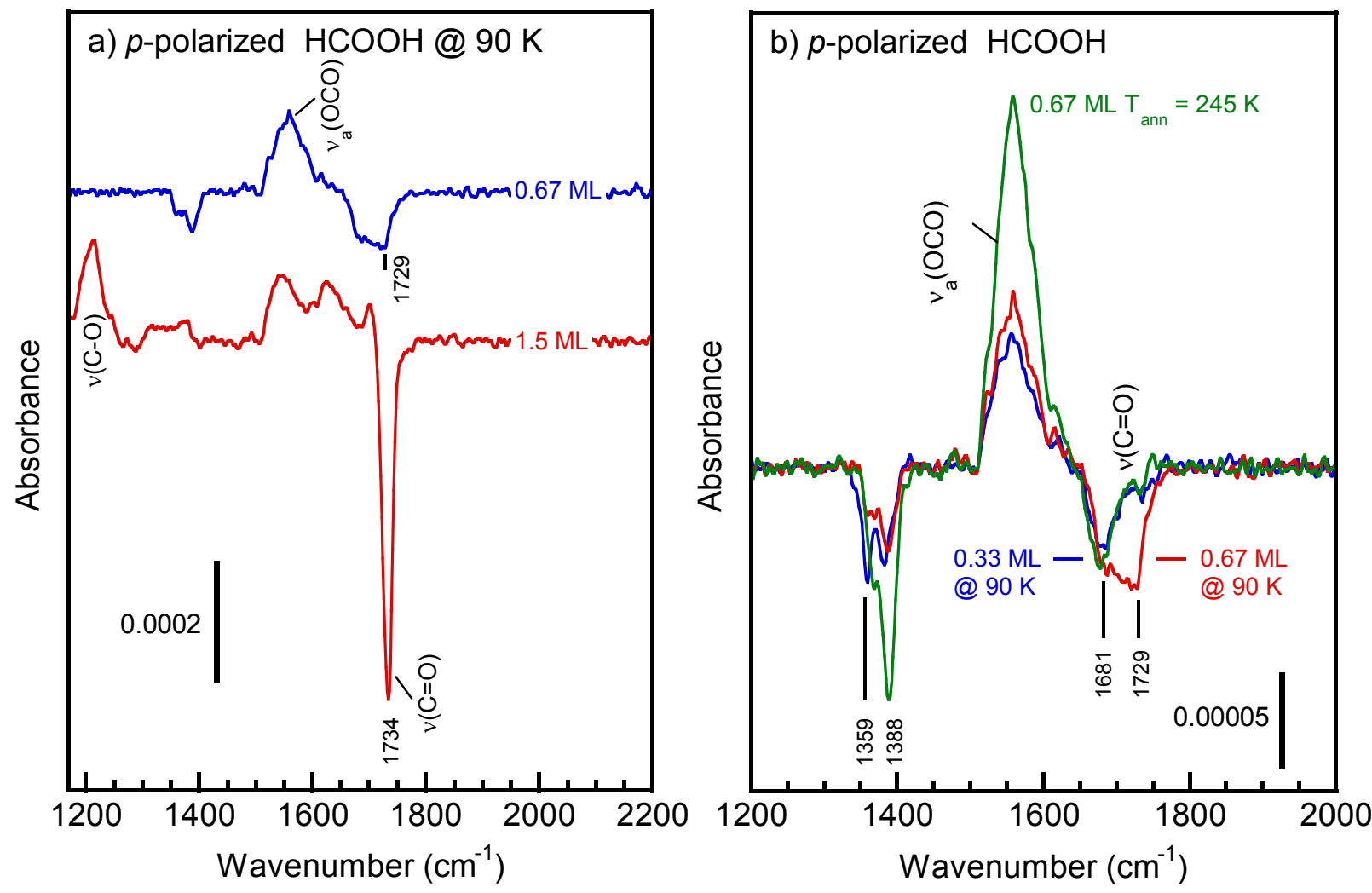

Figure S7. a) IRAS spectra in p-polarized mode of 0.67 and $1.5 \mathrm{ML} \mathrm{HCOOH}$ adsorbed on $\mathrm{TiO}_{2}(101)$ at $90 \mathrm{~K}$ (blue and red traces correspondingly). b) IRAS spectra in p-polarized mode of 0.33 and $0.67 \mathrm{ML} \mathrm{HCOOH}$ adsorbed on $\mathrm{TiO}_{2}(101)$ at $90 \mathrm{~K}$ (blue and red traces correspondingly) and of $0.67 \mathrm{ML} \mathrm{HCOOH}$ adsorbed on $\mathrm{TiO}_{2}(101)$ at $90 \mathrm{~K}$ and annealed at $245 \mathrm{~K}$ (green trace). 

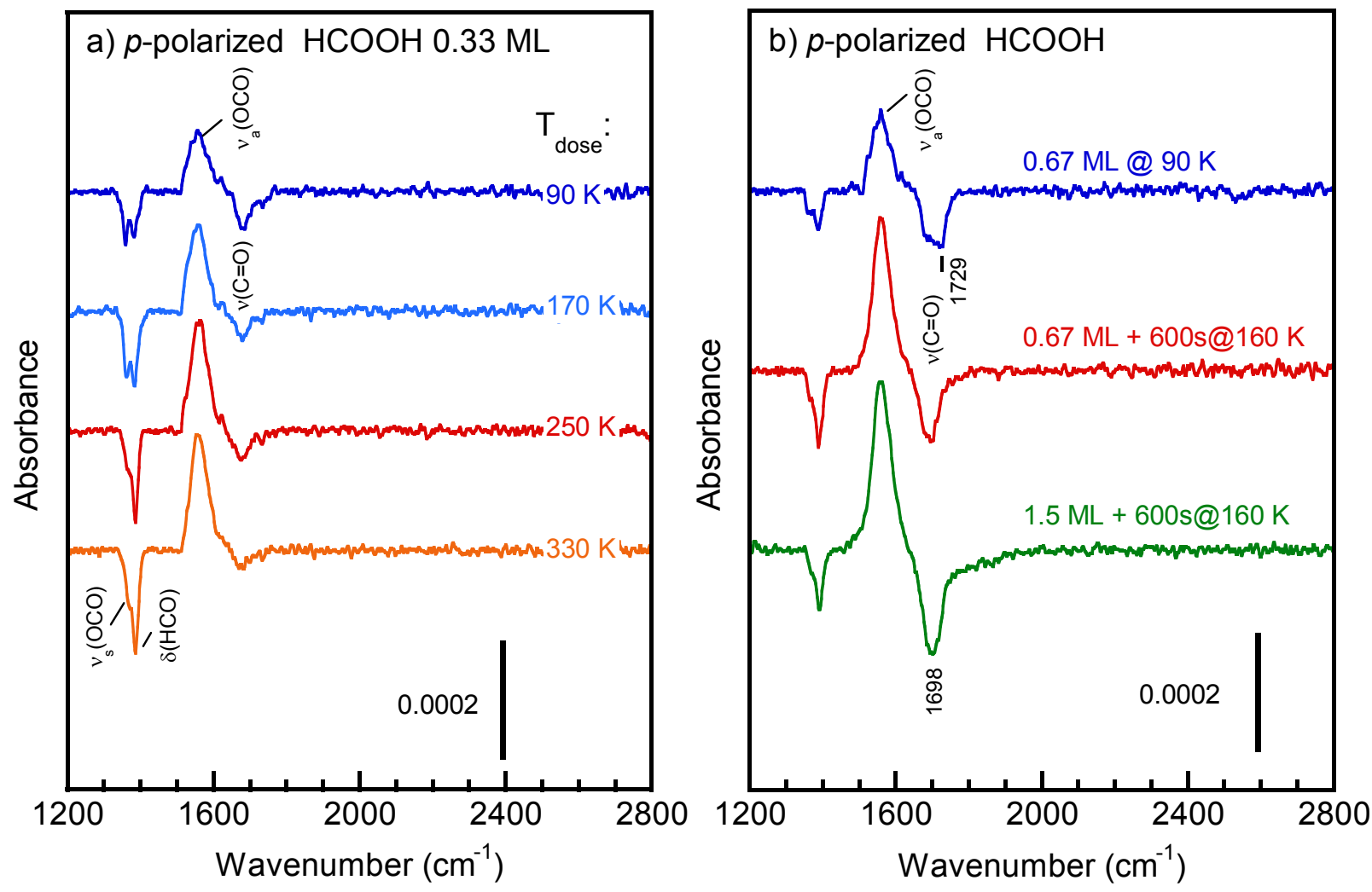

Figure S8. a) IRAS spectra in the p-polarized mode for $0.33 \mathrm{ML} \mathrm{HCOOH}$ adsorbed on $\mathrm{TiO}_{2}(101)$ at different temperatures. b) IRAS spectra in p-polarized mode of the $\mathrm{HCOOH}$ saturated monolayer coverage: $0.67 \mathrm{ML}$ dosed at $90 \mathrm{~K}$ (upper blue trace), same annealed 600s at $160 \mathrm{~K}$ (middle red trace), and $1.5 \mathrm{ML}$ dosed at $90 \mathrm{~K}$ and annealed $600 \mathrm{~s}$ at $160 \mathrm{~K}$ (lower green trace). 

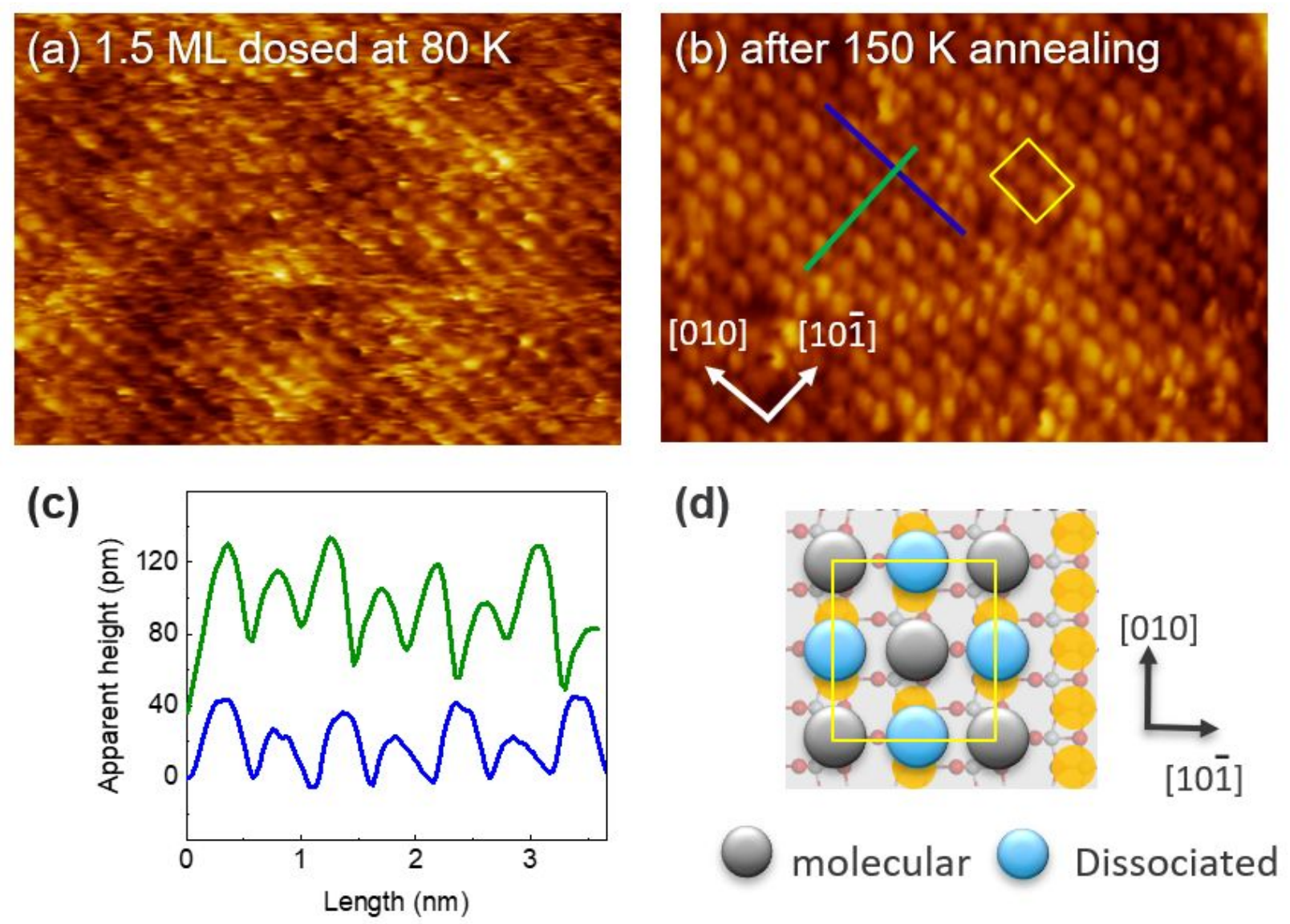

Figure S9. Scanning tunneling microscopy images after the adsorption of $1.5 \mathrm{ML}$ at $80 \mathrm{~K}$ (a), and after additional annealing to $150 \mathrm{~K}$ (b). The line scans along the [010] (blue) and [101] (green) directions (c) illustrating the local periodicities that are schematically shown in (d). 


\section{References}

1.Hayashi, S.; Umemura, J.; Kato, S.; Morokuma, K., Ab Initio Molecular Orbital Study on the Formic Acid Dimer. J Phys Chem 1984, 88, 1330-1334.

2.Faubel, M.; Kisters, T., Non-Equilibrium Molecular Evaporation of Carboxylic Acid Dimers. Nature 1989, 339, 527-529.

3.Farfan, P.; Echeverri, A.; Diaz, E.; Tapia, J. D.; Gomez, S.; Restrepo, A., Dimers of Formic Acid: Structures, Stability, and Double Proton Transfer. J Chem Phys 2017, 147, 044312. 